\title{
The role of palliative radiotherapy in bladder cancer: a narrative review
}

\author{
Sophie E. M. Raby ${ }^{1}$, Peter Hoskin ${ }^{2,3}$, Ananya Choudhury ${ }^{1,2}$ \\ ${ }^{1}$ Department of Clinical Oncology, The Christie NHS Foundation Trust, Manchester Academic Health Science Centre, Manchester, UK; ${ }^{2}$ Division \\ of Cancer Science, School of Medical Sciences, Faculty of Biology, Medicine and Health, University of Manchester, Manchester Academic Health \\ Sciences Centre, Manchester, UK; ${ }^{3}$ Mount Vernon Cancer Centre, Northwood, Middlesex, UK \\ Contributions: (I) Conception and design: None; (II) Administrative support: None; (III) Provision of study materials or patients: None; (IV) \\ Collection and assembly of data: None; (V) Data analysis and interpretation: None; (VI) Manuscript writing: All authors; (VII) Final approval of \\ manuscript: All authors. \\ Correspondence to: Dr. Sophie E. M. Raby. Department of Clinical Oncology, The Christie NHS Foundation Trust, Manchester Academic Health \\ Science Centre, Manchester, UK. Email: sophie.raby@nhs.net.
}

\begin{abstract}
Bladder cancer is the $6^{\text {th }}$ most common cancer worldwide and contributes significant excess mortality and morbidity. It often presents at a late stage when it is incurable, and the prognosis is poor. The local symptoms of bladder cancer-including haematuria, dysuria, frequency, nocturia and pain, have significant effects on quality of life and may require frequent inpatient admissions. As a palliative treatment, radiotherapy can be uniquely useful in providing targeted long term symptomatic control, although this must be balanced against the potential of causing toxicity. A variety of radiotherapy protocols have been developed for managing these symptoms. The results of several studies show that radiotherapy delivered in a hypofractionated regime (21 Gy in 3 fractions) can provide relief of these symptoms within a few weeks. Other commonly used regimes include 35 Gy in 10 fractions, 30 Gy in 5 fractions, a once weekly 36 Gy in 6 fractions, and a single 8 Gy fraction. In the palliative setting symptom resolution lasts for the majority of the patients remaining lifespan. Benefit is particularly clear for symptomatic haematuria and in these patients even single doses may provide rapid benefit. To maximise benefit from radiotherapy, studies are urgently needed to better estimate the prognosis of patients presenting with bladder cancer.
\end{abstract}

Keywords: Bladder cancer; radiotherapy; dysuria; frequency; haematuria

Submitted Jul 01, 2020. Accepted for publication Oct 05, 2020.

doi: 10.21037/apm-20-1347

View this article at: http://dx.doi.org/10.21037/apm-20-1347

\section{Introduction}

Bladder cancer is the $6^{\text {th }}$ most commonly occurring cancer worldwide. Each year there are over 550,000 new cases of bladder cancer and more than 200,000 deaths (1). Bladder cancer often presents at a late incurable stage and predominantly affects a more elderly population many of whom are unfit for radical treatment. Unfortunately prognosis is poor in this patient population and palliative treatment is therefore the mainstay of their oncological management (2). Significant tumour associated symptoms are common in these patients. Debilitating symptoms associated with local effects of bladder cancer can be split into three categories; haematuria, lower urinary tract symptoms (LUTS) (dysuria, urinary frequency and nocturia) and pain. All can cause significant detriment to quality of life requiring frequent inpatient admissions and the use of supportive medications associated with their own specific toxicities.

Palliative cancer care shifts the focus away from curing the disease and moves towards improving these symptoms and maintaining quality of life. For bladder cancer palliative treatment may consist of chemotherapy, immunotherapy, radiotherapy and close supportive medical care to alleviate 
symptom burden. Unfortunately uptake of appropriate palliative management in bladder cancer, in particular the utilisation of radiotherapy is often poor $(3,4)$.

Radiotherapy is a uniquely useful adjunct-when it is appropriately delivered-in providing long-term control of the symptoms of bladder cancer. It can be delivered rapidly, in requiring little of the patient's time, it is minimally invasive and can provide rapid and prolonged remission of symptoms. However as an intervention with the potential to cause toxicity, and where symptom control may take a time to materialize, the choice of which patients will derive overall benefit can be challenging $(5,6)$. Worryingly there is evidence that these difficulties have led to a deficit in the limiting of patient access to access of potentially curative treatment regimes for bladder cancer (4).

In advanced muscle invasive bladder cancer (MIBC) radiotherapy is commonly delivered to the whole bladder volume due to concerns regarding localising the lesion, changes in position between radiotherapy doses and the multifocal nature of many bladder cancers. The aim of this treatment is to relieve symptoms specific to the local disease but may also provide some temporary local control of disease. Although radiotherapy represents a more targeted option than systemic chemotherapy, side effects are common $(7,8)$, with basic techniques. Most significant toxicity comes from irradiation of adjacent bowel and bladder. Radiotherapy techniques have improved significantly over recent years. The majority of palliative radiotherapy is now given following $3 \mathrm{D}$ imaging to optimise identification of the target volume sand surrounding at organs at risk (OARs) minimising side effects from dosing to other local organs. The risks of toxicity must be balanced against the potential benefits in alleviating tumour specific symptoms. This is particularly important in these patients who are often elderly and frail.

Radiotherapy regimes used for MIBC pelvic radiotherapy vary widely and are influenced by healthcare system, patient and tumour factors. Hypofractionation allows the total dose of radiotherapy to be given over a shorter period of time. This has socio-economic advantages of limiting the impact on the patient and the radiotherapy department. With hypofractionation the risk of late toxicity ( $>6$ months from treatment) is theoretically increased however given that the median overall survival (OS) for palliative MIBC is 7.5 months, this is of limited concern (7). Reassuringly in the curative setting hypofractionated bladder radiotherapy has not been shown to increase late toxicity $(9,10)$.

The evidence for the efficacy of hypofractionated palliative regimes for bladder cancer is supported by the MRC BA09 randomised control trial. In this study 500 patients were randomised 1:1 to receive either 35 Gy in 10 fractions or $21 \mathrm{~Gy}$ in 3 fractions (7). The study collected detailed information on symptoms related to bladder cancer and radiotherapy toxicity before and after treatment. No difference was demonstrated between the two arms for either toxicity, symptom relief or OS. A once weekly 6 fraction course has also been shown to be equivalent to the more traditional 35 Gy in 10 fractions regime and may provide a more manageable alternative for patients struggling with mobility (11). Given the limited life expectancy for this patient group minimising the burden of treatment is crucial and therefore the shorter 3 fraction regime should be preferred in all patients. We present the following article in accordance with the Narrative Review reporting checklist (available at http://dx.doi.org/10.21037/ apm-20-1347).

\section{Radiotherapy outcomes for specific bladder symptoms}

Haematuria affects nearly a third of patients with MIBC and can cause pain and symptomatic anaemia $(7,12)$. Frank haematuria with clot formation can result in painful retention necessitating catheterisation. In those patients unfit for surgical intervention definitive management options may include embolization or radiotherapy. Radiotherapy is effective for haemostasis in multiple malignancies. The mechanism by which it achieves haemostasis is poorly understood but may be due to destruction of tissue microvasculature and/or stimulation of platelet activation $(13,14)$.

The BA09 trial provides the best evidence of the efficacy of radiotherapy for haemostasis in bladder cancer. At baseline 167 of 494 patients had significant haematuria, with $20 \%$ suffering from continuous haematuria or haematuria with visible clots. Immediately following the completion of the radiotherapy regime over half of these patients experienced improvement in their haematuria with worsening symptoms were seen in less than $10 \%$. The rapid onset (within days to weeks) of haematuria relief has been confirmed by several other retrospective studies $(15,16)$. In the BA09 study the benefit continued to accrue and at 3 months post treatment, in those patients still alive, $88 \%$ had resolution of their haematuria which was prolonged with a median time to deterioration of symptoms of 9 months.

Retrospective reviews from several institutes have 
confirmed this finding. A retrospective review of data from 2 UK centres identified 241 patients (20\%) who received radiotherapy for haematuria and at 6 weeks posttreatment over half of patient symptoms had resolved (2). Multiple different dosing schedules were used and there was no clear benefit of one fractionation strategy over any other. In a series of 65 patients treated with a once weekly regime of 36/30 Gy in 6 fractions (11). Thirty-seven had haematuria and $92 \%$ had symptom resolution at 3 months. A recent study of a similar regime demonstrated haematuria improvement in $91 \%$ of patients that in the majority persisted for their remaining lives with a median haematuria free survival of 13 months (17).

Pain, dysuria, frequency and nocturia-collectively referred to as LUTS - are common complaints in patients with MIBC. In contrast to haematuria the outcomes from radiotherapy are more mixed. One reason for this is that it is likely that even small lesions are capable of causing bladder irritation and generating these symptoms. Therefore even significant reduction in tumour bulk may not be sufficient to alleviate symptoms. Furthermore radiotherapy can cause irritation of the lining of the bladder and may exacerbate LUTS in the short term.

Again the BA09 trial provides detailed information. At base line $36 \%$ of patients had nocturia, $16 \%$ had frequency (greater than 1-2 episodes hourly) and $44 \%$ of patients had dysuria (7). At 3 -month follow up $88 \%$ of surviving patients had relief of dysuria, $62 \%$ had relief of nocturia and $72 \%$ had relief of frequency. At early time points (1-2 weeks) the results are more mixed with improvement for each symptom in around $20 \%$ of patients but a worsening of symptoms in a third. There was no significant difference between the two radiotherapy regimes in the number of patients for whom LUTS improved or the duration for which they improved, which was an average of 9 months. Given the median OS of 9 months this suggests that the majority of patients who become symptom free will remain so for their remaining lifespan.

Similar benefits were seen in retrospective cohort studies. After 36/30 Gy in 6 fractions there was improvement of LUTS in $51 \%$ at 1 month (11). The length of symptom relief was an average of 7 months with a median OS of 9 months. This again suggests that benefit may be achieved for the majority of a patient's lifespan. Jose et al. treated 65 patients with the same hypofractionated weekly dosing regime and identified similar findings regarding symptom control with frequency controlled in $68 \%$ of patients and nocturia in $50 \%$. Dysuria was only controlled in $10 \%$ of the population however numbers were small (18). A more recent report of 58 symptomatic patients with the same palliative regime identified resolution of pain and dysuria in $100 \%$ of patients of frequency and nocturia in $67 \%$ (19).

In summary for haematuria benefit from radiotherapy occurs rapidly within days to weeks and is maintained over the remaining lifespan of the patient. Thus for macroscopic haematuria resulting in significant symptoms or need for transfusion a trial of hypofractionated radiotherapy is in most cases an appropriate intervention even for those with limited life expectancy. Though the results for LUTS are less impressive than seen for haematuria, for the majority of patient treatment with radiotherapy would be a useful adjunct. However given the increased time to onset of benefit and consideration of toxicity and prognosis are of greater importance than treating for LUTS alone.

\section{Side effects of palliative bladder radiotherapy}

Radiotherapy side effects may occur from the target organ or nearby structures. In the setting of palliative bladder radiation short-term side effects are of the most concern, as the majority of patient will in most cases not survive long enough to experience late toxicities. Key early toxicities in bladder radiotherapy include the worsening of LUTS and acute bowel toxicities including diarrhoea, PR bleeding and rectal pain (19,20).

In the BA09 trial whilst worsening of all urinary symptoms was seen for nearly a third of patients by the end of treatment, at 3 months this had improved with only $20 \%$ of patients having worsened nocturia or frequency and only $10 \%$ having worsened dysuria. The absence of a control group makes these figures difficult to interpret and this persistent worsening of symptoms may simply represent patients with non-responding disease rather than treatment toxicity. Acute bowel toxicity was also seen in a significant number of patients at the end of treatment. Symptoms of frequency and diarrhoea were present in $36 \%$ and $40 \%$ of patients respectively and rectal pain was worsened in $20 \%$. The majority of symptoms had resolved by 3 months. The previously discussed retrospective cohort studies largely confirm these findings $(20,21)$. In the series receiving 36/30 Gy in 6 fractions (Mclaren et al.), 18\% of the 65 patients treated experienced acute grade 3 urinary toxicity and $12 \%$ experienced acute RTOG grade 3 bowel toxicity (11). Scholten et al. found diarrhoea in $36 \%$ and frequency in $65 \%$ but none of the 69 patients developed acute G3 toxicity. 
Late toxicity of radiotherapy affecting the bowel and bladder can include late onset of LUTs, bowel frequency and tenesmus or the development of bowel strictures, frozen pelvis or severe reduction of bladder volume. In one small study of hypofractionated regime of 36 Gy in 6 fractions late toxicity, defined as persisting LUTs at 1 year in absence of measurable disease, were present in $25 \%$ of patients (4/16). Late bowel toxicity of grade 3 or 4 was only seen in a single patient (18). Similar results were seen in two further studies with similar fractionation schedule in which $15 \%$ and $9 \%$ of patients had low grade late urinary toxicity $(17,20)$. One series of 125 relatively good prognosis patients with T2-T3 disease having a 5 -year survival of $39 \%$ receiving 36 Gy in 6 fractions reported severe (grade 3 ) late side effects in only 6 patients. This provides some reassurance about the safety of more hypofractionated regimes particularly in the setting of later disease with poor prognosis. Improving radiotherapy techniques such as adaptive planning-in which the radiotherapy target volume is updated before each fraction and margins around the target may be reduced-could decrease these toxicities even further (22).

\section{Completion of radiotherapy and prediction tools for life expectancy in bladder cancer}

Appropriate patient selection is important for ensuring maximum benefit for frail patients. In the BA09 study rates of treatment drop out were low with only 15 of 500 patients failing to complete treatment (7). In the real world setting results are worse with up to $20 \%$ of patients failing to complete treatment or dying within 30 days of treatment completion (2). Similarly $12 \%(8 / 65)$ of patients in one retrospective study did not complete the assigned radiotherapy course due to toxicity or death (11). Patients in both retrospective studies were treated in some cases with longer regimes as compared to the regimes in BA09, which were over 3 days to 2 weeks, and this may explain the difference in failure rates.

Studies in the setting of other palliative treatments for malignancy have shown that clinician estimation of prognosis, particularly towards the end of life is frequently inaccurate and over estimates life expectancy (23). To improve on this multiple groups have attempted to develop prognostic models to identify those patients with very short survivals who may not benefit from palliative radiotherapy. Anorexia, ECOG PS, and the presence of liver metastases were identified as important factors linked very short survival (24). These studies have not been validated in external datasets and therefore their utility is not yet clear. Furthermore, bladder cancer made up only a small fraction of patients within the training datasets raising questions as to the applicability to this disease. In palliative bladder cancer many unique clinical parameters have been associated with poor outcomes including haemoglobin, PS, lymphopenia and stage $(2,25)$. Validation of a prognostic model to identify those with very short survival in bladder cancer is urgently needed. Bladder cancer affects a predominantly elderly population who are often frail and have multiple comorbidities. In such patients, performance status may be insufficient to capture the extent of frailty. More detailed frailty measures are emerging as alternatives to PS and may provide useful adjuncts in bladder cancer management $(26,27)$.

\section{Conclusions and summary}

In summary bladder radiotherapy provides excellent and rapid palliation of symptoms. Given the clear absence of benefit for both OS and symptom control from prolonged regimes, short hypofractionated treatment regimes are recommended. Benefit is particularly clear for symptomatic haematuria and in these patients even single doses may provide rapid benefit in those with poor prognosis. It is important to remember that denying patients access to radiotherapy towards the end of life may cause harm allowing troublesome symptoms to persist.

Going forwards multisite prospective studies of practice would be informative. Alongside a move towards routine collection of patient reported outcome measures, such studies will allow a more personalised approach to identifying those patients most likely to benefit from bladder radiotherapy.

Research selection: PubMed searches were performed using the terms: bladder cancer, radiotherapy, dysuria, frequency and haematuria for all study designs. Search results were reviewed for relevance.

\section{Acknowledgments}

AC and PJH are supported by the NIHR Manchester Biomedical Research Centre.

Funding: None.

\section{Footnote}

Provenance and Peer Review: This article was commissioned 
by the Guest Editors (Edward Chow and Candice Johnstone) for the series "Palliative Radiotherapy" published in Annals of Palliative Medicine. The article has undergone external peer review.

Reporting Checklist: The authors have completed the Narrative Review reporting checklist. Available at http:// dx.doi.org/10.21037/apm-20-1347

Conflicts of Interest: All authors have completed the ICMJE uniform disclosure form (available at http:// dx.doi.org/10.21037/apm-20-1347). The series "Palliative Radiotherapy" was commissioned by the editorial office without any funding or sponsorship. AC reports grants from National Institute of Health Research Manchester Biomedical Research Centre, grants from Cancer Research, UK, grants from Medical Research Council, UK, grants from Prostate Cancer, UK, grants from Bayer, UK, personal fees from Janssen Pharmaceutical, non-financial support from ASCO, grants and non-financial support from Elekta $\mathrm{AB}$, outside the submitted work. The other authors have no conflicts of interest to declare.

Ethical Statement: The authors are accountable for all aspects of the work in ensuring that questions related to the accuracy or integrity of any part of the work are appropriately investigated and resolved.

Open Access Statement: This is an Open Access article distributed in accordance with the Creative Commons Attribution-NonCommercial-NoDerivs 4.0 International License (CC BY-NC-ND 4.0), which permits the noncommercial replication and distribution of the article with the strict proviso that no changes or edits are made and the original work is properly cited (including links to both the formal publication through the relevant DOI and the license). See: https://creativecommons.org/licenses/by-nc-nd/4.0/.

\section{References}

1. Richters A, Aben K, Kiemeney L. The global burden of urinary bladder cancer: An update. Springer.

2. Ali A, Song Y, Mehta S, et al. Palliative Radiation Therapy in Bladder Cancer-Importance of Patient Selection: A Retrospective Multicenter Study. Int J Radiat Oncol Biol Phys 2019;105:389-93.

3. Hugar LA, Lopa SH, Yabes JG, et al. Palliative care use amongst patients with bladder cancer. BJU Int
2019;123:968-75.

4. Gray PJ, Fedewa S, Shipley W, et al. Use of Potentially Curative Therapies for Muscle-invasive Bladder Cancer in the United States: Results from the National Cancer Data Base. Eur Urol 2013;63:823-9.

5. Mottet N, Ribal MJ, Boyle H, et al. Management of bladder cancer in older patients: Position paper of a SIOG Task Force. J Geriatr Oncol 2020;11:1043-53.

6. Spencer K, Morris E, Dugdale E, et al. 30 day mortality in adult palliative radiotherapy - A retrospective population based study of 14,972 treatment episodes. Radiother Oncol 2015;115:264-71.

7. Duchesne GM, Bolger JJ, Griffiths GO, et al. A randomized trial of hypofractionated schedules of palliative radiotherapy in the management of bladder carcinoma: results of medical research council trial BA09. Int J Radiat Oncol Biol Phys 2000;47:379-88.

8. Lutz ST, Chow EL, Hartsell WF, et al. A review of hypofractionated palliative radiotherapy. Cancer 2007;109:1462-70.

9. James ND, Hussain SA, Hall E, et al. Radiotherapy with or without chemotherapy in muscle-invasive bladder cancer. N Engl J Med 2012;366:1477-88.

10. Hoskin PJ, Rojas AM, Bentzen SM, et al. Radiotherapy with concurrent carbogen and nicotinamide in bladder carcinoma. J Clin Oncol 2010;28:4912-8.

11. McLaren DB, Morrey D, Mason MD. Hypofractionated radiotherapy for muscle invasive bladder cancer in the elderly. Radiother Oncol 1997;43:171-4.

12. Ghahestani SM, Shakhssalim N. Palliative treatment of intractable hematuria in context of advanced bladder cancer: a systematic review. Urol J 2009;6:149-56.

13. Yarnold J, Brotons MC. Pathogenetic mechanisms in radiation fibrosis. Radiother Oncol 2010;97:149-61.

14. Cihoric N, Crowe S, Eychmüller S, et al. Clinically significant bleeding in incurable cancer patients: effectiveness of hemostatic radiotherapy. Radiat Oncol 2012;7:132.

15. Lacarrière $\mathrm{E}$, Smaali $\mathrm{C}$, Benyoucef $\mathrm{A}$, et al. The efficacy of hemostatic radiotherapy for bladder cancer-related hematuria in patients unfit for surgery. Int Braz J Urol 2013;39:808-16.

16. Tey J, Soon YY, Cheo T, et al. Efficacy of Palliative Bladder Radiotherapy for Hematuria in Advanced Bladder Cancer Using Contemporary Radiotherapy Techniques. In Vivo 2019;33:2161-7.

17. Dirix P, Vingerhoedt S, Joniau S, et al. Hypofractionated palliative radiotherapy for bladder cancer. Support Care 
Cancer 2016;24:181-6.

18. Jose CC, Price A, Norman A, et al. Hypofractionated Radiotherapy for Patients with Carcinoma of the Bladder. Clin Oncol (R Coll Radiol) 1999;11:330-3.

19. Kouloulias V, Tolia M, Kolliarakis N, et al. Evaluation of Acute Toxicity and Symptoms Palliation in a Hypofractionated Weekly Schedule of External Radiotherapy for Elderly Patients with Muscular Invasive Bladder Cancer. Int Braz J Urol 2013;39:77-82.

20. Scholten AN, Leer JW, Collins CD, et al. Hypofractionated radiotherapy for invasive bladder cancer. Radiother Oncol 1997;43:163-9.

21. Hafeez S, McDonald F, Lalondrelle S, et al. Clinical Outcomes of Image Guided Adaptive Hypofractionated Weekly Radiation Therapy for Bladder Cancer in Patients Unsuitable for Radical Treatment. Int J Radiat Oncol Biol Phys 2017;98:115-22.

22. Huddart R, McDonald F, Lewis R, et al. HYBRID -

Cite this article as: Raby SEM, Hoskin P, Choudhury A. The role of palliative radiotherapy in bladder cancer: a narrative review. Ann Palliat Med 2020;9(6):4294-4299. doi: 10.21037/apm20-1347
Evaluating New Radiation Technology in Patients with Unmet Needs. Clin Oncol (R Coll Radiol) 2013;25:546-8.

23. Chow E, Davis L, Panzarella T, et al. Accuracy of survival prediction by palliative radiation oncologists. Int $\mathrm{J}$ Radiat Oncol Biol Phys 2005;61:870-3.

24. Chow E, Abdolell M, Panzarella T, et al. Predictive Model for Survival in Patients With Advanced Cancer. J Clin Oncol 2008;26:5863-9.

25. Joseph N, Dovedi SJ, Thompson C, et al. Pre-treatment lymphocytopaenia is an adverse prognostic biomarker in muscle-invasive and advanced bladder cancer. Ann Oncol 2016;27:294-9.

26. Rockwood K, Mitnitski A. Frailty defined by deficit accumulation and geriatric medicine defined by frailty. Clin Geriatr Med 2011;27:17-26.

27. Rolfson DB, Majumdar SR, Tsuyuki RT, et al. Validity and reliability of the Edmonton Frail Scale. Age Ageing 2006;35:526-9. 